\title{
Electrophysiological manifestations of non-penetrating cardiac trauma
}

\author{
KIM M FOX, EDWARD ROWLAND, DENNIS M KRIKLER, \\ HUGH H BENTALL, JOHN F GOODWIN \\ From the Division of Cardiovascular Disease, Royal Postgraduate Medical School, \\ Hammersmith Hospital, London
}

SUMMARY Electrophysiological disturbances were observed in four cases of non-penetrating cardiac trauma. Ventricular tachycardia occurred both as an early and late complication in three, responding to medical treatment in two; it was cured by cryoablation in the other case. The fourth patient developed an isolated conduction defect associated with anteroseptal myocardial infarction, the coronary arteries and left ventricular function being normal.

Non-penetrating injuries to the chest may affect the heart in several ways, including electrophysiological disturbance, in particular cardiac arrhythmia. ${ }^{12}$ Ventricular tachycardia poses a particular problem as it is usually incapacitating and may prove fatal. Though medications may control these arrhythmias, pacing or surgery may be required. ${ }^{3}$ We report the electrophysiological consequences of non-penetrating trauma to the heart in four patients: three had ventricular tachycardia including one with a resistant arrhythmia that required operation; the remaining patient developed an isolated conduction defect.

Received for publication 12 September 1979

\section{Case 1}

A 25-year-old man had suffered from recurrent palpitation associated with chest pain and dizziness for five years. During an episode an electrocardiogram disclosed ventricular tachycardia (Fig. 1); after cardioversion there were changes consistent with old anterior infarction (Fig. 2). At the age of 7 years a horse had kicked his chest, throwing him 20 metres. Examination and routine investigations including chest $x$-ray were normal. Left ventricular angiography showed an apical aneurysm (Fig. 3), but the coronary arteries were normal. Despite propranolol, verapamil, and disopyramide, the

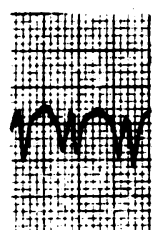

Fig. 1 Case 1 : electrocardiogram during tachycardia.
I

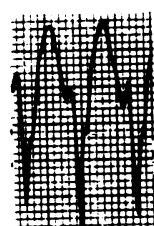

II

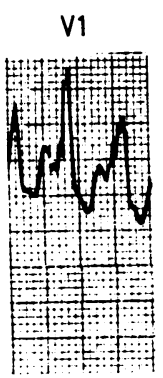

V2
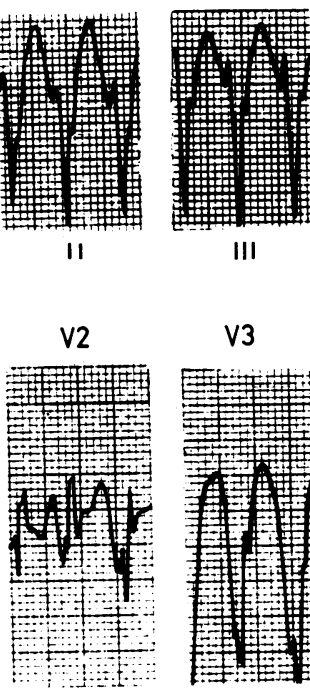

458
III

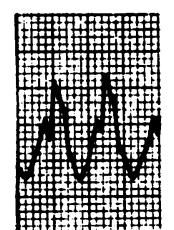

aVR

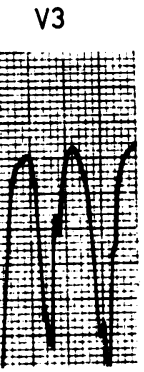

VL

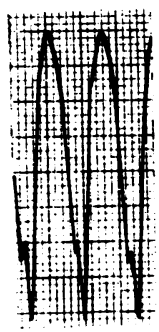

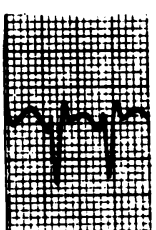

aVL

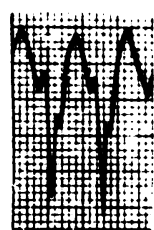

aVF 

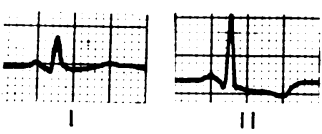

II
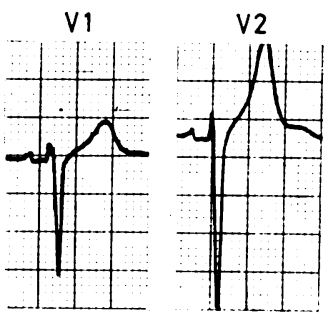

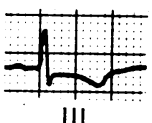

III

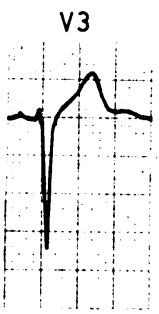

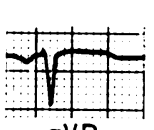

aVR

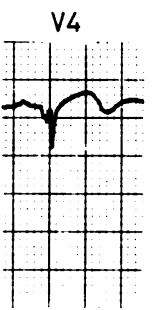

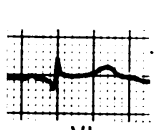

aVL

V5

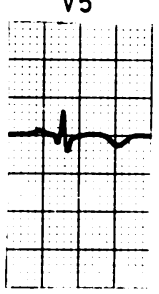

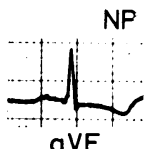

V6

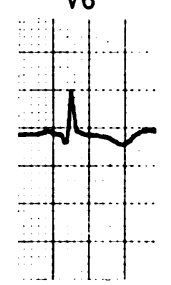

Fig. 2 Case 1: electrocardiogram in sinus rhythm showing changes of old anterior infarction. tachycardia recurred. An electrophysiological study ${ }^{4}$ disclosed no abnormality nor could tachycardia be induced, but pacing was not attempted from the left ventricle.

At operation, a small ventricular aneurysm was found adjacent to the anterior descending branch of the left coronary artery and related to the second diagonal branch; apart from a few fibrous adhesions the heart and coronary arteries were normal. On epicardial mapping with hand-held bipolar and tripolar electrodes the signals, together with atrial and ventricular reference electrograms, were recorded simultaneously with leads I, II, III, and aVF, using an Elema Mingograf 81 direct writing

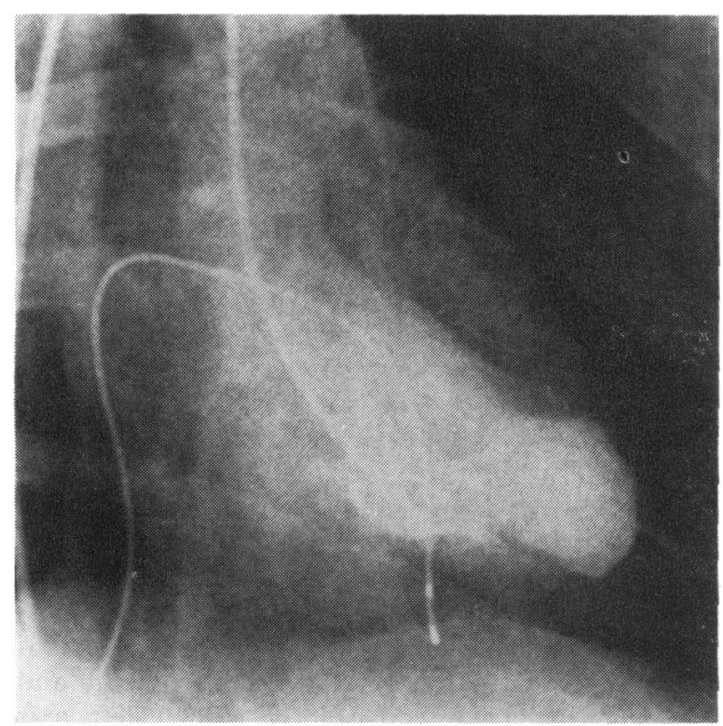

Fig. 3 Case 1 : left ventricular angiogram (right anterior oblique) showing an anteroapical aneurysm; there is a pacing wire in the right ventricle. recorder. During sinus rhythm activation was abnormal in the region of the aneurysm: epicardial potentials occurred up to $130 \mathrm{~ms}$ after the reference ventricular electrogram and well after the QRS complex (Fig. 4A). At the lower edge of the aneurysm the initial depolarisation during the QRS complex (Fig. 4B) was followed by an afterpotential ${ }^{5}$ and a subsequent ventricular extrasystole with the same configuration as seen during ventricular tachycardia. The relation between the reference and local potentials during the extrasystole was identical to that during tachycardia.

Ventricular tachycardia occurred spontaneously or could be initiated by two ventricular extrastimuli delivered through the ventricular reference electrode. Epicardial activation was earliest along the inferior border of the aneurysm and preceded the onset of the QRS complex by $20 \mathrm{~ms}$ (Fig. 4C); activation spread across the epicardial surface towards the base of the heart, again being slow along the left border of the aneurysm (Fig. 4D).

The left and inferior margin of the aneurysm was cryoablated ${ }^{6}$ and though conduction remained slow over the centre of the aneurysm during sinus rhythm, it proved impossible to reinitiate the tachycardia. The aneurysm was then plicated. The postoperative course was uncomplicated and he has been free from palpitation for two years.

\section{Case 2}

A 31-year-old male electrician developed chest pain and shortness of breath immediately after lifting an extremely heavy electrical cable $(200 \mathrm{~kg})$ and carrying it 200 metres. He was admitted to hospital and found to have ventricular tachycardia at a rate of 160 a minute. Intravenous practolol and oral digoxin had no immediate effect and the arrhythmia stopped after 23 hours. The subsequent 


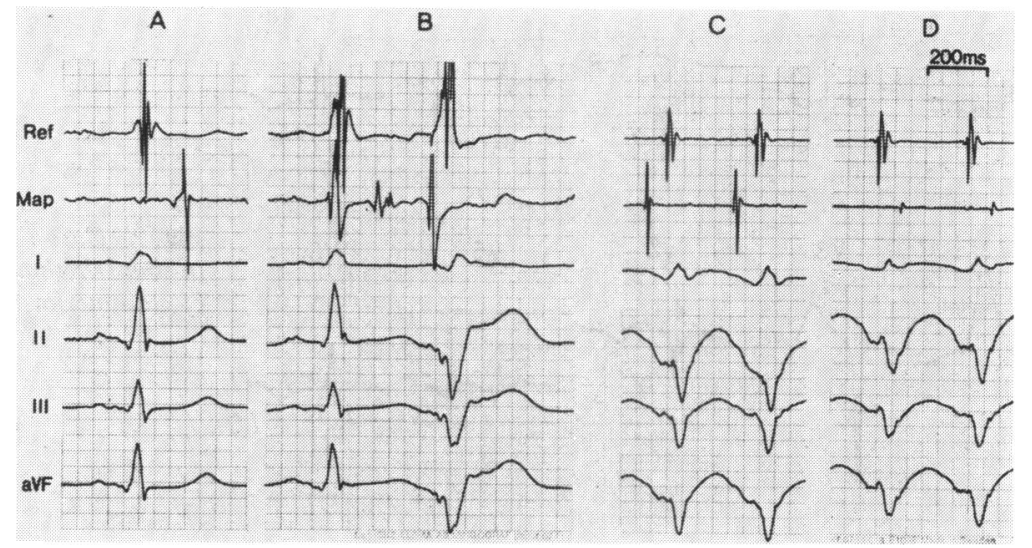

Fig. 4 Case 1 : Examples of the activation sequences recorded during epicardial mapping, paper speed $100 \mathrm{~mm} / \mathrm{s}$. Each panel shows an epicardial electrogram recorded from a fixed reference point $2 \mathrm{~cm}$ cranial to the aneurysm (Ref), an epicardial electrogram recorded with the moveable electrode (Map), and leads I, II, III, and aVF. (A) Sinus rhythm, Map recorded from the centre of the aneurysm. Slow conduction is shown by the delayed local electrogram after the $Q R S$. (B) Sinus rhythm, Map recorded from the lower border of the aneurysm. A local electrogram during the narrow $Q R S$ complex is followed by a low amplitude afterpotential and a third electrogram at the onset of a ventricular extrasystole which has a similar QRS morphology to that seen during ventricular tachycardia. (C) Map during ventricular tachycardia showing early local electrograms from the area of earliest epicardial activation, close to the lower pole of the aneurysm. (D) Map during ventricular tachycardia showing delayed local electrogram from the area of latest epicardial activation, at the left upper border of the aneurysm.

electrocardiogram in sinus rhythm showed transient $S T$ and $T$ wave abnormalities in the inferior leads. Over the next six months he continued to suffer from tachycardia which always ceased spontaneously. He was admitted to the Hammersmith Hospital for further investigation. There were no cardiac abnormalities on examination and the resting electrocardiogram was normal, with a normal QTc interval and no abnormalities of repolarisation or evidence of ventricular hypertrophy. Chest $x$-ray and echocardiogram were both normal. Intracardiac electrography demonstrated normal atrioventricular nodal function both anterogradely and retrogradely, with no evidence of an accessory pathway or of atrioventricular nodal reentry. On slow release quinidine (Kinidin Durules) he was free from tachycardia for two years so treatment was stopped and he has remained asymptomatic for the subsequent two years.

\section{Case 3}

A 46-year-old man had been completely well until the age of 31 when he developed a severe constricting chest pain while lifting a heavy weight. An electrocardiogram recorded three days later showed inferior myocardial infarction. He remained well until three years later when he was admitted to hospital with palpitation and shortness of breath but the electrocardiogram showed sinus rhythm.
He then remained asymptomatic until one year before referral when he developed recurrent paroxysmal ventricular tachycardia. Though termination was often spontaneous, cardioversion was required 13 times in that year; there was no response to various prophylactic agents (quinidine, procainamide, disopyramide, digoxin, and betareceptor antagonists). On admission he looked fit and had no clinical abnormalities. The resting electrocardiogram showed old inferior infarction; chest $x$-ray was normal. Echocardiogram showed an isovolumic contraction abnormality and the posterior wall of the left ventricle was hypokinetic. Angiography showed conspicuous inferior and apical dyskinesia with normal coronary arteries. On amiodarone $(200 \mathrm{mg} /$ day) he has been completely free from palpitation for two years.

\section{Case 4}

A fit 31-year-old male engineer had normal routine electrocardiograms performed for his employment on oil rigs, including a tracing in May 1977 (Fig. 5A). In June 1977 a steel plate fell on to his chest and he lost consciousness briefly. Electrocardiograms recorded 10 days after the accident, and subsequently, have consistently shown left axis deviation and loss of $\mathbf{R}$ waves in the anterior chest leads consistent with anterior myocardial infarction (Fig. 5B). Physical examination was normal and 
coronary arteriography and left ventricular angiography performed one year after the accident were likewise normal. He remains free of symptoms.

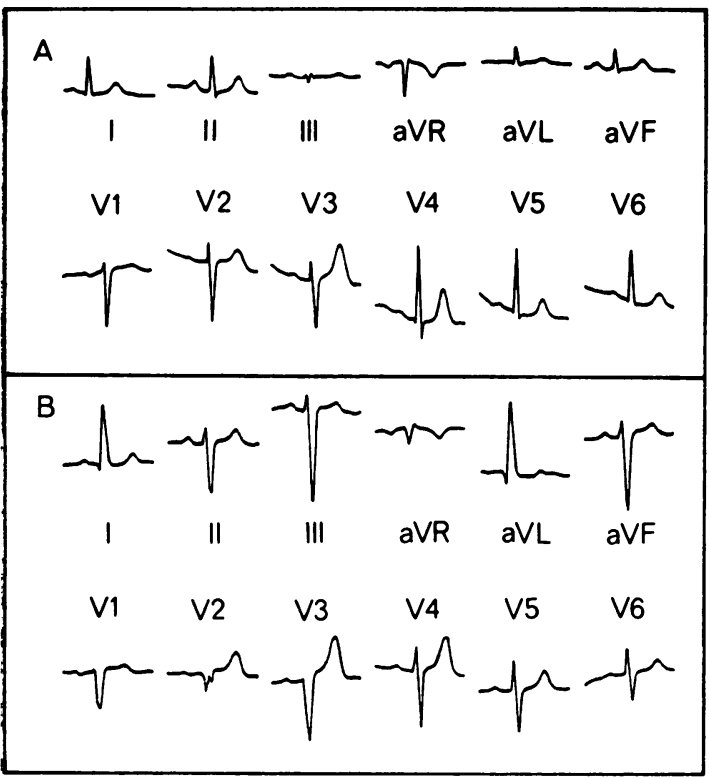

Fig. 5 Electrocardiograms, case 4: (A) one month before injury: normal tracing; (B) 10 days after injury there is left axis deviation as well as loss of $R$ waves in the anterior chest leads.

\section{Discussion}

Several mechanisms may be responsible for cardiac damage by non-penetrating trauma to the chest, including sudden acceleration or deceleration, direct compression by the sternum, or sudden increase in intrathoracic pressure ${ }^{7}$; the last seems applicable to cases 2 and 3 . It can be impossible to distinguish between myocardial contusion and direct injury to a coronary artery. A wide variety of electrophysiological manifestations have been seen hours, months, or even years after the injury, including complete heart block and supraventricular and ventricular tachycardia. ${ }^{8-10}$ Ventricular tachycardia is the most dramatic consequence and may result in syncope or even death; unfortunately, its treatment is often difficult and though there are now many useful antiarrhythmic agents, alternative forms of treatment may occasionally be needed. 51112

Acute myocardial infarction with subsequent development of a left ventricular aneurysm, as in case 1 , is a well-recognised consequence of nonpenetrating trauma to the chest. ${ }^{13} 14$ Coronary arteriography has only rarely been performed after traumatic myocardial infarction, the coronary arteries usually being normal; as mentioned, it is unclear whether the infarct results from myocardial contusion or from direct injury to a coronary artery which then recanalises. ${ }^{715}$ The long interval between the trauma and the development of the tachycardia in cases 1 and 3 is noteworthy.

We could not induce tachycardia during the electrophysiological investigation in case 1 ; a pacing electrode was not inserted into the left ventricle and because of the origin of the tachycardia close to the aneurysm, the right ventricular electrode was almost certainly too distant to allow us to demonstrate the features later seen at intraoperative mapping. At operation re-entrant tachycardia could be provoked from the left border of the aneurysm, with slowing of conduction between normal and abnormal myocardium which could produce changes in refractoriness. At operation it was not feasible to examine the response of the delayed afterpotential to critically timed premature ventricular beats or to assess the pattern of intramyocardial conduction with plunge electrodes.

The successful response to cryoablation after epicardial mapping emphasises the potential value of this approach to the treatment of refractory ventricular tachycardia. This emerges from observations that long-term survivors who had undergone aneurysmectomy for recurrent ventricular tachycardia relapsed after operation when epicardial mapping and cryoablation were not performed ${ }^{16}$; the arrhythmias might have persisted because the initiating site located in the margin of the aneurysm was not destroyed. ${ }^{16}{ }^{17} \mathrm{We}$ believe that this is the first occasion in which cryoablation has been used for the treatment of this form of tachycardia. However, medical management may well succeed (cases 2 and 3 ).

In case 4 the development of electrocardiographic changes consistent with acute myocardial infarction and left axis deviation after chest trauma poses several questions, and raises medicolegal aspects. Left axis deviation in this context implies a disturbance of conduction in the left bundle-branch, perhaps by the effects of contusion. Despite the normal coronary arteries and left ventricular function, it is possible that further electrophysiological manifestations may appear later, including a greater degree of heart block ${ }^{8}$ or, as in case 1, ventricular tachycardia, even without an aneurysm. ${ }^{5}$ With greater awareness of the possible electrophysiological effects of injury to the heart, other cases similar to ours will be recognised, and a history of trauma to the chest should be sought in otherwise unexplained arrhythmias and conduction disturbances. 


\section{References}

${ }^{1}$ Barber $\mathrm{H}$. The effects of trauma, direct or indirect, on the heart. $Q \mathcal{F}$ Med $1944 ; 13: 137-67$.

${ }^{2}$ Parmley LF, Manion WC, Mattingly TW. Nonpenetrating traumatic injury to the heart. Circulation 1958; 18: 371-96.

${ }^{3}$ Gallagher JJ, Oldham HN, Wallace AG, Peter RH, Kassell J. Ventricular aneurysm with ventricular tachycardia. Report of a case with epicardial mapping and successful resection. Am $\mathcal{F}$ Cardiol 1975; 35: 696700.

${ }^{4}$ Curry PVL. Fundamentals of arrhythmias: modern methods of investigation. In: Krikler DM, Goodwin JF, eds. Cardiac arrhythmias, the modern electrophysiological approach. London, Philadelphia, Toronto: WB Saunders, 1975: 39-80.

${ }^{5}$ Fontaine G, Guiraudon G, Frank R, Vedel J, Grosgogeat Y, Cabrol C. Modern concepts of ventricular tachycardia. The value of electrocardiological investigations and delayed potentials in ventricular tachycardia of ischemic and nonischemic etiology (31 operated cases). Eur $\mathcal{F}$ Cardiol 1978; 8: 565-80.

${ }^{6}$ Gallagher JJ, Sealy WC, Anderson RW, et al. Cryosurgical ablation of accessory atrioventricular connections. A method for correction of the pre-excitation syndrome. Circulation 1977; 55 : 471-9.

${ }^{7}$ De Feyter PJ, Roos JP. Traumatic myocardial infarction with subsequent normal coronary arteriogram. Eur f Cardiol 1977; 6: 25-31.

${ }^{8}$ Sims BA, Geddes JS. Traumatic heart block. Br Heart f 1969; 31: 140-2.

${ }^{9}$ Bharati S, Chervony A, Gruhn J, Rosen EM, Lev M. Atrial arrhythmias related to trauma to sino-atrial node. Chest 1972; 61: 331-5.
${ }^{10}$ Symbas PN. Cardiac trauma. Am Heart $\mathcal{f} 1976$; 92: 387-96.

${ }^{11}$ Ritter ER. Intractable ventricular tachycardia due to ventricular aneurysm with surgical cure. Ann Intern Med 1969; 71: 1155-7.

${ }^{12}$ Ritchie JL, Hammermeister KE, Kennedy JW. Refractory ventricular tachycardia and fibrillation in a patient with the prolapsing mitral leaflet syndrome: successful control with overdrive pacing. Am $\mathcal{F}$ Cardiol 1976; 37: 314-6.

${ }^{13}$ Warburg E. Myocardial and pericardial lesions due to non-penetrating injury. $\mathrm{Br}$ Heart $\mathcal{f} 1940$; 2: 271-80.

${ }^{14}$ Levy H. Traumatic coronary thrombosis with myocardial infarction. Arch Intern Med 1949; 84: 261-76.

${ }^{15}$ Harthorne JW, Kantrowitz PA, Dinsmore RE, Sanders C. Traumatic myocardial infarction-report of a case with a normal angiogram. Ann Intern Med 1967; 66: 341-4.

${ }^{16}$ Sami M, Chaitman BR, Bourassa MG, Charpin D, Chabot M. Long term follow up of aneurysmectomy for recurrent ventricular tachycardia or fibrillation. Am Heart f 1978; 96: 303-8.

${ }^{17}$ Guiraudon G, Fontaine G, Frank R, Escande G, Etievent $P$, Cabrol $C$. Encircling endocardial ventriculotomy: a new surgical treatment for life-threatening ventricular tachycardias resistant to medical treatment following myocardial infarction. Ann Thorac Surg 1978; 26 : 438-43.

Requests for reprints to $\mathrm{Dr}$ Dennis Krikler, Division of Cardiovascular Disease, Hammersmith Hospital, London W12 0HS. 